\title{
Background of Gravitational Waves Generated by Astrophysical Sources
}

\author{
José C. N. de Araujo and Oswaldo D. Miranda \\ Divisão de Astrofísica - Instituto Nacional de Pesquisas Espaciais, \\ Avenida dos Astronautas 1758 - São José dos Campos - 12227-010 SP - Brazil
}

(Received on 23 October, 2005)

We have recently shown [1] that the dimensionless amplitude of the stochastic background of gravitational waves (GWs) produced by an ensemble of sources is given by

$$
h_{\mathrm{BG}}^{2}=\frac{1}{v_{\mathrm{obs}}} \int h_{\mathrm{single}}^{2} d R,
$$

where $h_{\text {single }}$ is the dimensionless amplitude produced by an event that generates a signal with observed frequency $v_{\mathrm{obs}}$; and $d R$ is the differential rate of production of GWs.

Eq.(1) is in fact a shortcut to the calculation of stochastic background of GWs. An interesting characteristic of this equation is that it is not necessary to know in detail the energy flux of the GWs produced at each frequency. If the characteristic values for the dimensionless amplitude and frequency are known and the event rate is given it is possible to calculate the stochastic background of GWs produced by an ensemble of sources.

In particular, for the case of a background produced by an ensemble of black holes, the differential rate reads

$$
d R_{\mathrm{BH}}=\dot{\rho}_{*}(z) \frac{d V}{d z} \phi(m) d m d z
$$

where $\dot{\rho}_{*}(z)$ is the star formation rate density (hereafter $\mathrm{SFRD})$; in $\left.\mathrm{M}_{\odot} \mathrm{yr}^{-1} \mathrm{Mpc}^{-3}\right), d V$ is the comoving volume element and $\phi(m)$ is the initial mass function (IMF). In a few words, the $\phi(m) d m$ represents the number of stars per unit mass in the interval $[m, m+d m]$. The normalization of the IMF is obtained through the relation

$$
\int_{\mathrm{m}_{\mathrm{l}}}^{\mathrm{m}_{\mathrm{u}}} m \phi(m) d m=1
$$

where it is usually considered that $\mathrm{m}_{1}=0.1 \mathrm{M}_{\odot}$ and $\mathrm{m}_{\mathrm{u}}=125 \mathrm{M}_{\odot}$ (see, e.g., Ref. [1]).

We present here an alternative and in fact a more robust derivation of Eq.(1). We start from the specific flux received in GWs at the present epoch as (see, in particular, Eq. (15) in Ref.[2] and section 12.1 in Ref.[3])

$$
F_{v}\left(v_{\mathrm{obs}}\right)=\int \frac{l_{v}}{4 \pi d_{\mathrm{L}}^{2}} \frac{d v}{d v_{\mathrm{obs}}} d V
$$

where

$$
l_{v}=\frac{d L_{v}}{d V}
$$

is the comoving specific luminosity density (given, e.g, in $\mathrm{erg} \mathrm{s}^{-1} \mathrm{~Hz}^{-1} \mathrm{Mpc}^{-3}$ ), which obviously refers to the source frame.

As discussed in Refs.[2, 3], the above equations are valid to estimate a stochastic background radiation received on Earth independent of its origin. In the present paper $l_{v}$ can be written as follows

$$
l_{v}=\int \frac{d E_{\mathrm{GW}}}{d v} \dot{\rho}_{*}(z) \phi(m) d m,
$$

where $d E_{\mathrm{GW}} / d v$ is the specific energy of the source. Note that in the above equation $\dot{\rho}_{*}(z)$ refers to the source frame, therefore, there is not any putative $(1+z)$ factor related to time dilation.

Thus, the flux $F_{v}\left(v_{\text {obs }}\right)$ received on Earth reads

$$
F_{\mathrm{v}}\left(\mathrm{v}_{\mathrm{obs}}\right)=\int \frac{1}{4 \pi d_{\mathrm{L}}^{2}} \frac{d E_{\mathrm{GW}}}{d v} \frac{d v}{d v_{\mathrm{obs}}} \dot{\rho}_{*}(z) \phi(m) d m d V .
$$

Using Eq.(2) it follows that

$$
F_{\mathrm{v}}\left(\mathrm{v}_{\mathrm{obs}}\right)=\int \frac{1}{4 \pi d_{\mathrm{L}}^{2}} \frac{d E_{\mathrm{GW}}}{d v} \frac{d v}{d v_{\mathrm{obs}}} d R_{\mathrm{BH}}
$$

Note that in the above equation, what multiplies $d R_{\mathrm{BH}}$ is nothing but the specific energy flux per unity frequency (in, e.g., $\operatorname{erg~cm}^{-2} \mathrm{~Hz}^{-1}$ ), i.e.,

$$
f_{v}\left(v_{\mathrm{obs}}\right)=\frac{1}{4 \pi d_{\mathrm{L}}^{2}} \frac{d E_{\mathrm{GW}}}{d v} \frac{d v}{d v_{\mathrm{obs}}}
$$

(see, e.g., Ref.[4]).

On the other hand, the specific energy flux per unit frequency for GWs is given by Ref.[5]

$$
f_{\mathrm{v}}\left(\mathrm{v}_{\mathrm{obs}}\right)=\frac{\pi c^{3}}{2 G} h_{\mathrm{BH}}^{2}
$$

Also, the spectral energy density, the flux of GWs, received on Earth, $F_{\mathrm{v}}$, in erg $\mathrm{cm}^{-2} \mathrm{~s}^{-1} \mathrm{~Hz}^{-1}$ can be written from Refs. $[6,7]$ as

$$
F_{\mathrm{v}}\left(\mathrm{v}_{\mathrm{obs}}\right)=\frac{\pi c^{3}}{2 G} h_{\mathrm{BG}}^{2} \mathrm{v}_{\mathrm{obs}} .
$$

From the above equations one obtains

$$
h_{\mathrm{BG}}^{2}=\frac{1}{v_{\mathrm{obs}}} \int h_{\mathrm{BH}}^{2} d R_{\mathrm{BH}},
$$

which is nothing but the Eq. (1). 


\section{Acknowledgments}

JCNA would like to thank the Brazilian agency $\mathrm{CNPq}$ for partial support (grant 303868/2004-0).

[1] J.C.N. de Araujo, O.D. Miranda, and O.D. Aguiar, Phys. Rev. D 61, 124015 (2000).

[2] A. J. Farmer and E. S. Phinney, Mon. Not. R. Astron. Soc. 346, 1197 (2003).

[3] J. A. Peacock, in Cosmological Physics (Cambridge University Press, Cambridge, England, 1999).

[4] V. Ferrari, R. Schneider, and S. Matarrese, Mon. Not. R. Astron. Soc. 303, 258 (1999).
[5] B. J. Carr, Astron. Astrophys. 89, 6 (1980).

[6] D. H. Douglass and V. G. Braginsky, in General Relativity: An Einstein Centenary Survey, edited by S. W. Hawking and W. Israel (Cambridge University Press Cambridge, England, 1979), p. 90.

[7] D. Hils, P. L. Bender, and R. F. Webbink, Astrophys. J. 360, 75 (1990). 